\title{
Exact diagonalization study of the two-dimensional $t-J$-Holstein model
}

\author{
A. Dobry, A. Greco, S. Koval and J. Riera \\ Instituto de Física Rosario, Consejo Nacional de Investigaciones Científicas y Técnicas \\ y Departamento de Física, Universidad Nacional de Rosario, \\ Av. Pellegrini 250, 2000-Rosario, Argentina.
}

(Received today)

\begin{abstract}
We study by exact diagonalization the two-dimensional $t-J$-Holstein model near quarter filling by retaining only few phonon modes in momentum space. This truncation allows us to incorporate the full dynamics of the retained phonon modes. The behavior of the kinetic energy, the charge structure factor and other physical quantities, show the presence of a transition from a delocalized phase to a localized phase at a finite value of the electron-phonon coupling. We have also given some indications that the $e$-ph coupling leads in general to a suppression of the pairing susceptibility at quarter filling.
\end{abstract}

PACS Numbers: 71.27.+a, 71.38.+i, 74.20.Mn, 74.25.Kc

Typeset Using REVTEX 
The $t-J$ model is one of the simplest models proposed to understand the physics of strongly correlated electronic systems. [1] In particular, the two-dimensional $t-J$ model has been extensively studied in the context of high- $\mathrm{T}_{c}$ superconductivity since it contains the essential low-energy physics of the $\mathrm{CuO}_{2}$ planes present in the cuprates. Many magnetic, transport and superconducting properties of these materials have been described by this model. [2] However, since this model contains only electronic degrees of freedom, it cannot account for other properties, like the isotope effect, which requires the coupling of electron and phonon degrees of freedom. The inclusion of phonon degrees of freedom could also be relevant to describe electronic transport properties. Moreover, given the basic nature of the $t-J$ model, it is of general interest to study the effects of electron-phonon (e-ph) coupling in the framework of strongly correlated systems.

Our starting point is the two-dimensional (2D) $t-J$ model. Exact diagonalization studies of this model on the square lattice have given strong unbiased indications of $\mathrm{d}_{x^{2}-y^{2}}$ superconductivity at quarter filling $(n=0.5)$ in the vicinity of phase separation. [3, th These indications are absent in the one-band 2D Hubbard model, which is the most basic model of strongly correlated electronic systems.

The $e$-ph coupling is incorporated in the Hamiltonian by a Holstein term in which an Einstein phonon with frequency $\omega$ is coupled linearly to the local electronic density. The Holstein model, which describes uncorrelated electrons with this type of $e$-ph coupling, has been studied to understand the relationship between superconductivity and Peierls chargedensity-wave (CDW) formation. Quantum Monte Carlo simulations have been done in one dimension [5] and more recently in two dimensions. [6, [7] In one dimension, it was shown in Ref. [5] that the Holstein model undergoes a transition to a CDW state above a critical value of the $e$-ph coupling in the case of spinless fermions, but the ordered state sets in for arbitrary value of the coupling for the case of spin- $\frac{1}{2}$ fermions.

The total Hamiltonian including both electron and phonon degrees of freedom is:

$$
H=-t \sum_{<i, j>, \sigma}\left(\tilde{c}_{j \sigma}^{\dagger} \tilde{c}_{i \sigma}+\tilde{c}_{i \sigma}^{\dagger} \tilde{c}_{j \sigma}\right)+J \sum_{<i, j>}\left(\mathbf{S}_{i} \cdot \mathbf{S}_{j}-\frac{1}{4} n_{i} n_{j}\right)
$$




$$
+\omega \sum_{i}\left(b_{i}^{\dagger} b_{i}+\frac{1}{2}\right)+g \sum_{i}\left(b_{i}^{\dagger}+b_{i}\right) n_{i}
$$

where $\tilde{c}_{i \sigma}^{\dagger}$ is an electron creation operator at site $i$ with spin $\sigma$ with the constraint of no double occupancy, $n_{i}=n_{i, \uparrow}+n_{i, \downarrow}$ is the electron number operator, and $b_{i}^{\dagger}$ is a phonon creation operator at site $i$. Here $t$ is the hopping parameter, which we choose as the scale of energies, $J$ is the antiferromagnetic exchange interaction and $g$ is the $e$-ph coupling. We adopt $\omega=1.0$ throughout.

The model defined by (1) is usually called the $t-J$ - Holstein model. Both this model and the Hubbard-Holstein model have been studied with a variety of analytical and numerical methods. 88.9] Most exact diagonalization studies have been performed so far for static phonons, in the so-called adiabatic or frozen phonon approximation. [9] The dynamics of phonons are therefore missed in these studies. Thus, their results for many physical quantities that require the inclusion of the full quantum nature of phonon degrees of freedom are questionable.

The purpose of this report is to extend the exact diagonalization techniques used for the pure $t-J$ model, in particular the Lanczos method, to the $t-J$-Holstein model, preserving the dynamics of phonons. This extension cannot be done without further approximation because the bosonic part of the Hilbert space is infinite dimensional. A truncation of the Hilbert space of phonons in real space was proposed in Ref. [10]. In this approach, only two states are allowed for each mode. Although the choice of these states in terms of coherent states is pressumably optimal, [11] it would be extremely hard to check the validity of the resulting variational space. Alternatively, we propose in this report a truncation in momentum space of phonons, retaining a small number of phonon modes. This approximation allows us to take enough boson states for each of the retained phonon modes so that the physical quantities can converge to the exact values for the most interesting region of parameters. [12] The exact results thus obtained for a restricted set of phonon modes in momentum space could be used to check approximate methods like e.g. the one proposed in Ref. [10]. 
In order to present our approach, we rewrite the last two terms of Eq. (1) in momentum space as:

$$
\begin{array}{r}
\left.H_{p h}=\sum_{\mathbf{q}} \omega(\mathbf{q})\left(b_{\mathbf{q}}^{\dagger} b_{\mathbf{q}}+\frac{1}{2}\right)\right) \\
H_{e-p h}=\sum_{\mathbf{q}} g(\mathbf{q})\left(b_{\mathbf{q}}^{\dagger}+b_{-\mathbf{q}}\right) \sum_{j} n_{j} e^{i \mathbf{q} \cdot \mathbf{r}_{j}}
\end{array}
$$

Actually, Eq. (2) is a generalization of the respective terms in (11) because we have allowed a $\mathbf{q}$ dependence in the constants $\omega$ and $g$. In fact, this $\mathbf{q}$ dependence leads to a more realistic e-ph coupling for many materials. In the case of the cuprates, starting from the three band Hubbard model in the presence of a planar breathing-like phonon, an effective $e$-ph coupling was derived with the form of Eq. (2) where $\omega(\mathbf{q})$ and $g(\mathbf{q})$ are maximum at $\mathbf{q}=(\pi, \pi)$. 13. The different strength of the e-ph coupling for different phonon modes in momentum space provides a physical justification of our truncation scheme. That is, we only keep in the phonon Hilbert space those states of the phonons with largest $e$-ph coupling strength. In the following, and for the sake of simplicity, we consider a $g$ independent of the phonon momentum.

Let us consider the case in which only states of the phonon $\mathbf{q}=(\pi, \pi)$ are contained in the phonon part of the Hilbert space. The exact ground state of the Hamiltonian (四) and (2) can be easily obtained for the case in which $J=t=0$, for an arbitrary electronic state $\left\{n_{i, \sigma}\right\}$. This ground state is minimum when all electrons, if possible, are located in one of the two sublattices of the square lattice. Then, for finite $t$ and $J$, a $(\pi, \pi)$ CDW order is expected when $g$ is large enough. In Fig. 1, we show the ground state energy vs. number of states of this phonon for the tilted $\sqrt{10} \times \sqrt{10}$ square lattice with $n=0.6, J=1.0$, and several values of $g$. The convergence to the exact result is quite fast in the range $0.0<g<0.55$. Between $0.55<g<0.6$ the transition to the above mentioned CDW region occurs. This convergence rate is typical of many exchange couplings and fillings studied.

In the first place, we study various physical quantities in the $\sqrt{10} \times \sqrt{10}$ cluster at $n=0.6$ as a function of $g$. Their behaviour, shown in Fig. 2, indicate a transition at $g=g_{c}$ from a phase which is essentially the same as in the pure $t-J$ model $(g=0)$ to a radically 
different phase which is essentially the one corresponding to $t=J=0$. The first phase is usually called the "delocalized" phase, and the second one, the "localized" phase. The kinetic energy is shown in Fig. 2(a). It has a slow variation for $0<g<g_{c}$. For $g>g_{c}$, the kinetic energy decreases rapidly to zero indicating a strong electron localization. We discuss further this feature below. The transition to the localized region can be seen quite clearly in the behavior of the $(\pi, \pi)$ CDW structure factor defined as:

$$
\chi_{c}(\mathbf{q})=\frac{1}{N} \sum_{i, j}<n_{i} n_{j}>e^{i \mathbf{q} \cdot\left(\mathbf{r}_{\mathbf{i}}-\mathbf{r}_{\mathbf{j}}\right)}
$$

with $\mathbf{q}=(\pi, \pi)$, and in the occupation number of phonons $n_{p h}=<b_{\mathbf{q}}^{\dagger} b_{\mathbf{q}}>$, shown in Figs. 2(b) and 2(c) respectively. The presence of two phases can also be observed in other quantities like the spin structure factor at $(\pi, \pi)$, shown in Fig. 2(d).

The transition to the CDW state is similar to that observed in the spinless Holstein model by Hirsch and Fradkin. [5] These authors derived an effective $\tilde{t}-V$ model, where $V$ is an effective nearest neighbor (nn) repulsion, to describe this transition. $\tilde{t}$ is a renormalized hopping $(\tilde{t} \ll t)$ that contains the effect of increased effective mass of the charge carriers. The fact that in our results the kinetic energy goes to zero inmediately after the transition is because most of the electrons are located in one of the two sublattices as it was indicated above and this state is deeply buried energetically. This feature should disappear if states of additional phonon modes are included in the Hilbert space. In order to check this scenario we have performed calculations for the same cluster and filling by retaining three phonon modes in momentum space. The three phonon modes are $(\pi, \pi)$, and $(4 \pi / 5,2 \pi / 5)$ and $(-4 \pi / 5,-2 \pi / 5)$ which belong to the closest shell to $(\pi, \pi)$. In this case, we obtained a convergence of four digits in the kinetic energy with 4 states per phonon mode for $g \leq 0.3$, and 5 states per phonon mode for $0.4 \leq g \leq 0.6$. The results for the kinetic energy for various values of $J$ are shown in Fig. 3(a). We have also reproduced for comparison the curves for $J=1.0$ and $J=3.0$ obtained by the phonon $(\pi, \pi)$ alone. Since the phononic Hilbert space is enlarged with respect to the previous one, the energy of the system in the region where phonons dominate should be lowered, and a simple variational argument then 
explains why the transition occurs at a smaller $g_{c}$. As expected, the kinetic energy decreases more slowly after the transition. The transition is smoother and the influence of the $e$-ph coupling is noticeable at much smaller $g$. For fixed $J$ and $g$, the kinetic energy is smaller than the corresponding value obtained for the case of a single mode, indicating a larger increase of the effective mass of the carriers. In Fig. 3(b) it can be seen that the charge structure factor at $\mathbf{q}=(3 \pi / 5,-\pi / 5)$, which is the largest at $g=0$ in the binding region $(J \geq 2.0)$, is suppressed by $g$ and the structure factor at $\mathbf{q}=(4 \pi / 5,2 \pi / 5)$ is enhanced and becomes dominant for $g>g_{c}$. Thus, the localized region is an incommensurate CDW order.

As in Ref. [5], it is reasonable to assume that our electron-phonon model can be described by an effective, purely electronic, $t-J-V$ model. In this model, the effective nn repulsion $V$ acts against binding of holes on nearest neighbor sites, thus suppressing pairing but then also preventing phase separation (PS). The outcome of these competing effects is not easily predictable and numerical calculations have in fact indicated an enhancement of pairing correlations. [14] In the $t-J$-Holstein model, we have an additional competing effect, i.e. the renormalization of the mass of the carriers. In order to determine the result of these effects, we have computed the kinetic energy and the pairing susceptibility for the $4 \times 4$ lattice at quarter filling. The static pairing susceptibility is defined as:

$$
\chi_{S C}=\frac{1}{N} \sum_{i, j}<\Delta_{i}^{\dagger} \Delta_{j}>
$$

where

$$
\Delta_{i}=\sum_{\mu} g_{\mu} c_{i \uparrow} c_{i+\mu \downarrow}
$$

where the sum extends over the nearest neighbors of site $i$ and $g_{\mu}$ are the form factors that determine the pairing symmetry. We report here results for the extended-s and $\mathrm{d}_{x^{2}-y^{2}}$ pairing symmetries.

We have considered only the phonon $(\pi, \pi)$ in the calculation. The exact diagonalization of the $4 \times 4$ cluster at quarter filling, was performed with momentum $(0,0)$ and d-wave rotational symmetry. The spin reversal symmetry was also taken into account to reduce 
the dimension of the electronic part of the Hilbert space. The energy converges with approximately 20 states for the phonon mode. Let us first examine the results for the kinetic energy, shown in Fig. $1(\mathrm{a})$. The general behavior is similar to that found for the $\sqrt{10} \times \sqrt{10}$ lattice. However, a slight upward change of the kinetic energy for $J=3.5$ and $J=4$, for large $g$ can be seen. In the pure $t-J$ model, $J=3.5$ is precisely at the PS border. [3] The influence of the effective repulsive interaction is then to shift the PS border to larger $J$.

Finally, in Fig. $₫($ (b) we show results for the pairing susceptibility. For the pure $t-J$ model, as shown in Ref. [3], the $\mathrm{d}_{x^{2}-y^{2}}$ pairing dominates over the extended-s pairing and its maximum occurs for $J=3$, i.e. just before the PS border. The effect of $e$-ph coupling is in general to suppress pairing. Only for $J=3.5$ and $J=4$ and large $g$, consistently with the behavior of the kinetic energy, an enhancement of the pairing susceptibility can be observed. If more phonon modes are added, taking into account the results previously discussed, it is expected that the effective nn repulsion turns out to be weaker than for the one phonon case. Besides, the mass renormalization is expected to be stronger. Thus, the net effect would be an overall suppression of the pairing correlations and susceptibility.

In summary, we have studied using exact diagonalization the $2 \mathrm{D} t-J$-Holstein model by retaining only few phonon modes in momentum space. Near quarter filling, the behavior of the kinetic energy, the charge structure factor, and other physical quantities, show the presence of a transition from a delocalized phase to a localized phase at a finite $g_{c}$. This value of $g_{c}$ decreases when the number of phonon modes is increased. We have also given some indications that the $e$-ph coupling leads in general to a suppression of the pairing susceptibility at quarter filling. Although preliminary, these are among the first results for a 2D electron-phonon model which incorporates the full dynamics of the phonon field and could be relevant to physically realistic situations. 


\section{REFERENCES}

[1] See e.g. Correlated electron systems, edited by V. J. Emery (World Scientific, Singapore, 1993).

[2] E. Dagotto, Rev. Mod. Phys. 66, 763 (1994).

[3] E. Dagotto and J. Riera, Phys. Rev. Lett. 70, 682 (1993).

[4] Y.Ohta, T.Shimozato, R. Eder, and S. Maekawa, Phys. Rev. Lett. 73, 324 (1994).

[5] J. E. Hirsch and E. Fradkin, Phys. Rev. B 27, 4302 (1983).

[6] R. Scalettar, N. Bickers, and D. Scalapino, Phys. Rev. B 40, 197 (1989).

[7] R. Noack, D. Scalapino, and R. Scalettar, Phys. Rev. Lett. 66, 778 (1991); M. Vekić, R. M. Noack, and S. R. White, Phys. Rev. B 46, 271 (1992).

[8] U. Trapper et al., Z. Phys. B 93, 465 (1994).

[9] H. Röder, H.Fehske, and H. Buttner, Phys Rev. B 47, 12420 (1993).

[10] R. Fehrenbacher, Phys. Rev. B 49, 12230 (1994); R. Fehrenbacher, unpublished.

[11] This variational space has also been used to study quantum lattice fluctuations by $\mathrm{H}$. Zheng, D. Feinberg, and M. Avignon, Phys. Rev. B 39, 9405 (1989). See also, H. Zheng, M. Avignon, and K. H. Bennemann, Phys. Rev. B 49, 9763 (1994).

[12] Exact results were obtained by taking up to 40 states for each of the two relevant phonon modes in a three site cluster by J. Mustre de Leon et al., Phys. Rev. Lett. 68, 3236 (1992).

[13] K. von Szczepanzki and K. Becker, Z. Phys. B 89, 327 (1992).

[14] E. Dagotto and J. Riera, Phys. Rev. B 46, 12084 (1992). 


\section{FIGURES}

FIG. 1. The ground state energy $\mathrm{E}_{0}$ as a function of the number of states of the $(\pi, \pi)$ phonon for the $\sqrt{10} \times \sqrt{10}$ cluster with $n=0.6, J=t=\omega=1.0$, and several values of $g$.

FIG. 2. (a) Kinetic energy, (b) charge structure factor $\chi_{c}(\pi, \pi),(\mathrm{c})$ phonon occupancy, and (d) spin structure factor $\chi_{s}(\pi, \pi)$, as a function of the $e$-ph coupling, obtained by exact diagonalization for the $\sqrt{10} \times \sqrt{10}$ cluster with $n=0.6, t=\omega=1.0$, and several values of $J$. The symbols used in (b), (c) and (d) are the same as in (a).

FIG. 3. (a)The kinetic energy as a function of $g$ for the $\sqrt{10} \times \sqrt{10}$ cluster with $n=0.6$, $t=\omega=1.0$, and several values of $J$. Full lines correspond to the set of $(\pi, \pi),(4 \pi / 5,2 \pi / 5)$ and $(-4 \pi / 5,-2 \pi / 5)$ phonons. Results for the $(\pi, \pi)$ phonon alone (dashed lines) are included for comparison. (b)The CDW structure factor at $(3 \pi / 5,-\pi / 5)$ (full lines) and at $(4 \pi / 5,2 \pi / 5)$ (dashed lines) as a function of $g$. The coupling constants are the same as in (a).

FIG. 4. (a) Kinetic energy, and (b) pairing susceptibility for $d_{x^{2}-y^{2}}$ (solid symbols) and extended-s (open symbols) symmetries, for the $4 \times 4$ lattice at quarter filling and $J=2.0$ (up triangles), $J=2.5$ (circles), $J=3.0$ (squares), $J=3.5$ (diamonds) and $J=4.0$ (down triangles). 